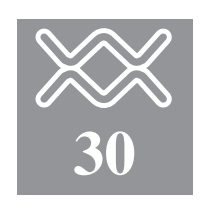

\title{
Ill catetere dii Tesio per emodialisi: 18 anni di evoluzione
}

\author{
F. Tesio \\ Dirigente Responsabile al 2003 \\ del Dipartimento di Medicina Interna e U.O. di Nefrologia e Dialisi \\ Azienda Ospedaliera S. Maria degli Angeli, Pordenone
}

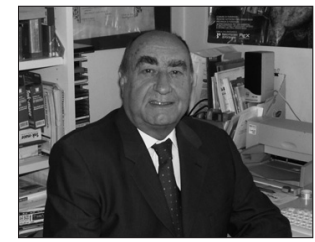

Ua pressoché scomparsa delle controindicazioni all'emodialisi (HD) in soggetti un tempo ritenuti intrattabili e lo sviluppo crescente nella richiesta di HD hanno contribuito in maniera sostanziale allo sviluppo della tecnologia del cateterismo venoso centrale per HD (1) sebbene la fistola artero-venosa di Cimino-Brescia continui a rappresentare il "golden standard" degli accessi vascolari. Dovendo ricorrere al cateterismo, dobbiamo però avere anche presente (2) che l'evidenza dimostra un aumento della morbilità e mortalità, maggior rischio di attuare dialisi inadeguate e un'alta percentuale di infezioni per i portatori di CVC longterm.

I CVC a lunga permanenza per emodialisi si sono nel tempo differenziati da quelli utilizzati per altre metodologie per ridurre le complicazioni ad essi legate. Al fine di rispondere efficacemente alle richieste delle attuali strategie dialitiche, oltre ai calibri adeguati sono evoluti prima dai tipi monolume a quelli bilume con più elevati e costanti flussi ematici e successivamente, seguendo la strada tracciata dai cateteri per dialisi peritoneale, divenendo tunnellizzabili nel sottocute per ridurre la contaminazione batterica pericannulare, la possibile dislocazione e la rimozione involontaria o accidentale.

Gli ultimi modelli offrono numerosi vantaggi in quanto sono facili da inserire in anestesia locale e permettono di iniziare, subito dopo l'inserimento, la seduta dialitica. Consentono un flusso ematico adeguato, riducono lo stress al paziente determinato dalle ripetute punture della FAV e, se correttamente gestiti, mantengono una buona funzionalità per molti anni.

L'incidenza di complicanze quali il malfunzionamento secondario ad errato posizionamento, kinking, trombosi, "fibrin sleeve" e sepsi nelle sue varie manifestazioni cliniche è da ritenere, anche se accettabile in relazione allo stato clinico dei portatori (3) passibile di miglioramento.

Nel 1986, Canard presentava un'idea originale di accesso ai vasi per HD (4): era costituito dall'impianto in vena giugulare di due cateteri invece di un unico catetere a due lumi. I due cateteri inseriti separatamente avevano dato risultati eccellenti pur essendo rappresentati da due drenaggi chirurgici in silastic adattati. Da questa idea abbiamo progettato, un sistema di accesso ai vasi tramite CVC a lunga permanenza denominato Catetere di Tesio e da allora assemblato in kit di al- lestimento (prodotto dalla ditta Medcomp, Harleysville, PA, USA). Rispetto al sistema di Canaud abbiamo riconsiderato il materiale componente i cateteri, il sistema di ancoraggio, la connessione esterna e gli accessori di accompagnamento onde semplificare le manovre di impianto. Questo kit utilizzabile per tutti gli accessi venosi centrali, è stato studiato particolarmente per l'incannulamento della vena giugulare interna (Fig. 1).

È ormai unanime l'accordo di evitare, quando possibile, la cateterizzazione della vena succlavia per l'elevata incidenza di complicazioni stenotiche che possono ostacolare il circolo venoso non solo dell'arto omolaterale e inficiare l'esito di successivi interventi di allestimento di una FAV. I danni di questa via di accesso sono stati dimostrati nel 1990 da Cimochowski (5) e poi nel 1991 da Schillinger (6) e dallo stesso Uldall (7) primo pioniere della via succlavia che ha deciso nel 1992 di sospendere la pratica dell'incannulazione della vena succlavia. Una ulteriore conferma è venuta nel 1995 da Konner (8): «Subclavian haemodialysis access: is still justified in 1995?». - Le due cannule flottanti separate che compongono il catetere riducono la possibilità di occlusione del catetere e permettono un flusso di sangue di $350-400 \mathrm{ml} / \mathrm{min}$. 


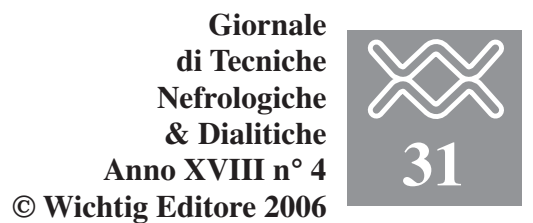

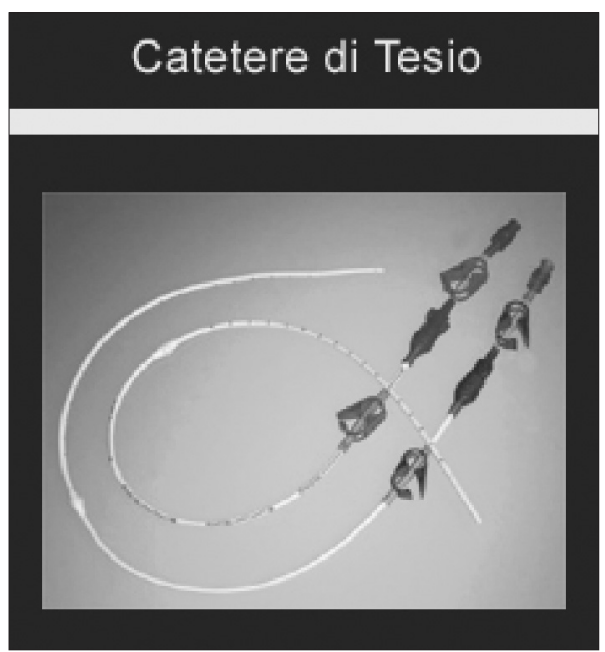

Fig. 1 - Cateteri Tesio ${ }^{\circledR}$ in silicone o poliuretano.

- La possibilità di inserire prima le cannule nei vasi e poi tunnellizzarle permette un loro esatto posizionamento in vena.

- Estensioni sostituibili che in caso di usura delle stesse possono essere cambiate evitando la sostituzione delle cannule.

- Disponibili in silicone (Tesio ${ }^{\circledR}$ Originale) o Bio-Flex ${ }^{\mathrm{TM}}$ (poliuretano).

- Cuffie in silicone nella versione in silicone e in dacron nella versione in Bio-Flex ${ }^{\mathrm{TM}}$.

- Nella versione in Bio-Flex ${ }^{\mathrm{TM}}$ sono disponibili modelli (anche lunghi 70 $\mathrm{cm}$ ) con cuffie posizionate a varia distanza dalla punta del catetere per una miglior adattabilità alla corporatura dei pazienti e per consentire l'inserimento delle cannule sul lato sinistro o preparare lunghi tragitti sottocute per incannulamenti della vena femorale e fuoriuscita in addome.

La tecnica d'inserimento del catetere Tesio ${ }^{\circledR}$ con procedura di tunnel retrogrado permette:

- Inserimento del catetere nel vaso prima della tunnellizzazione.

- Esatto posizionamento del catetere nella vena prima della tunnellizzazione e corretto posizionamento della punta del catetere e della cuffia. - Di usare estensioni sostituibili che in caso di usura delle stesse possono essere cambiate evitando la sostituzione del catetere.
Il disegno delle punte separate permette:

- Alti flussi con basse pressioni venose.

- Ridotto rischio di occlusione del catetere.

- Ricircolo inferiore all'1\%.

I risultati ottenuti (9) ci avevano fatto sperare di aver trovato una soluzione tale da proporre addirittura il sistema Tesio in alternativa alle FAV ma questi risultati, ottenuti con un'attenta monitorizzazione di pazienti selezionati e personale infermieristico estremamente motivati, non sono più stati raggiunti quando il sistema si è diffuso su larga scala. Anche se ha notevolmente ridotto le complicazioni e migliorata l'efficienza dialitica (10-13) rispetto ad altri cateteri "long-term", il catetere di Tesio deve rappresentare solo un ultimo accesso obbligato quando non è più possibile o consigliabile allestire una FAV. Messa in atto positivamente, è l'indicazione anche nei casi di emergenza quando si prevede di dover lasciare a permanenza il catetere per tempi superiori alle tre settimane.

\section{Considerazioni}

Le moderne strategie dialitiche ed altre metodiche correlate a una circolazione extracorporea del sangue richiedono, frequentemente, un flusso ematico elevato (>300 $\mathrm{ml} / \mathrm{min})$ non associato a ricircolo ematico (14). Per questi motivi abbiamo adottato l'idea di due cateteri introdotti separatamente: al contrario delle cannule a due lumi paralleli o coassiali, le due cannule indipendenti possono essere posizionate e distanziate nel modo più corretto per limitare il ricircolo mentre il diametro interno di $2 \mathrm{~mm}$ (o $3 \mathrm{~mm}$ nel catetere con calibro 12 Fr) permette gli alti flussi desiderati.

Abbiamo evitato di inserire i due cateteri attraverso un unico introduttore come suggerito da Powelson (10) per non perdere uno dei vantaggi della doppia cateterizzazione: se inseriamo le due cannule separatamente, avremo due lesioni della parete venosa di 3 $\mathrm{mm}$ di diametro, posizionate a distanza: la loro rimozione, quando dovuta, darà luogo a due piccole cicatrici disassate difficilmente causa di stenosi come avviene invece con i cateteri a due lumi, che hanno un calibro minimo di $6 \mathrm{~mm}$.

Altro vantaggio delle due cannule indipendenti, in caso di infezione o malfunzionamento di una delle due, è quello di poter continuare il programma emodialitico con il sistema monoago anche in caso di rimozione di uno dei due cateteri. Unico svantaggio per l'operatore che esegue l'intervento di incannulazione è la necessità di pungere due volte la vena ma, se l'intervento è effettuato sotto controllo ecografico, lo svantaggio risulta più teorico che reale.

La connessione con il raccordo esterno, che avviene dopo la tunnellizzazione delle cannule, facilita la puntura della vena che viene fatta direttamente per via percutanea al triangolo di Sedillot subito sopra il margine superiore della clavicola. La clavicola stessa poi regola il raggio di curvatura delle cannule evitando il rischio di kinking per la tunnellizzazione nel sottocute in avanti e in basso parallelamente allo sterno. Nel corso degli anni tutte le case produttrici di cateteri CVC a lunga permanenza si sono indirizzate verso l'accesso vascolare tramite la vena giugulare e l'utilità di avere un lungo tunnel sottocutaneo parallelo allo sterno: infatti propongono cateteri bilume con la curva periclavicolare già preformata (una delle complicazioni più frequenti con questi cateteri difficili da tunnellizzare era la strozzatura di una delle due vie ma resta lo svantaggio di un alto ricircolo ematico). Le più gravi complicazioni dei cateteri a lunga permanenza sono le infezioni e le ostruzioni trombotiche. Per ridurre $\mathrm{i}$ rischi di infezione e di attivazione dei fattori della coagulazione, abbiamo sfruttato due caratteristiche: una relativa alle modalità di posizionamento e una al materiale di composizione delle cannule.

Uno studio dei ricercatori dell'Istituto Oncologico di Aviano con il nostro gruppo (15) ci portava a ritenere che la colonizzazione microbica pericannulare era favorita dagli spostamenti "dentro-fuori" che i movimenti del 
braccio o la stessa pulsazione cardiaca imprimevano ai cateteri che avevano l'emergenza cutanea posizionata lateralmente rispetto alla sede di puntura della vena. Abbiamo pertanto ritenuto che il posizionamento migliore dell'exit-site dovesse trovarsi in avanti e in basso ad almeno $6 \mathrm{~cm}$ dalla clavicola dopo un percorso sottocute parallelo allo sterno. Il connettore esterno da collegare alle cannule in un secondo tempo dopo la loro fuoriuscita dalla cute facilita la loro tunnelizzazione. Era inoltre indispensabile che le normali difese dell'organismo non venissero inibite o comunque debilitate dalla presenza di un materiale scarsamente biocompatibile. Per una cateterizzazione permanente si richiede la stabilità chimica e una buona biostabilità, emocompatibilità e istocompatibilità. Dopo i primi anni di esperienza con il silicone standard, abbiamo scelto come materiale lo Spi-silicone in quanto, oltre ad avere le caratteristiche richieste, presenta una ridotta irregolarità della parete, tipica dei siliconi, ed è caratterizzato da un pretrattamento di infissione ionica che gli conferisce un valore di energia di superficie compresa fra i 20 e i 30 dynes per $\mathrm{cm}^{2}$ (16). Viene in tal modo prevenuta o quantomeno ridotta l'adesione alle pareti di piastrine, funghi, batteri e simili (17). Negli ultimi anni le miscele che compongono i poliuretani, hanno notevolmente migliorato le caratteristiche di stabilità chimica e biocompatibilità e pertanto hanno potuto essere preparati cateteri long-time con il vantaggio rispetto al silicone, di avere una parete più sottile con un migliore rapporto fra diametro interno ed esterno (a parità di diametro interno i cateteri occupano uno spazio inferiore nella vena). Fra i poliuretani di ultima generazione, ci siamo orientati verso un poliuretano che ci sembrava conciliare meglio le specifiche richieste e fatto preparare, in alternativa ai tradizionali cateteri in Spi-silicon, anche dei kit con cateteri in poliuretano Bio-Flex.

Dato che il flusso ematico idoneo alle moderne strategie dialitiche deve mantenersi costantemente alto, è stata prevista l'eventualità di poter con facilità sostituire una cannula eventual- mente malfunzionante dotando il catetere di un sistema di ancoraggio che consentisse la manovra. Per ancorare i cateteri abbiamo ideato un sistema interno che non necessita in prossimità dell'exit site di fili o altro materiale possibile causa di focus batterico. A seconda delle necessità, può essere costituito da un anello in spugna di dacron preposizionato sulla cannula in un punto che venga a trovarsi lungo il percorso sottocute (a imitazione dei cateteri per DP) o, come noi preferiamo, da un semplice rigonfiamento olivare della cannula. Questo secondo sistema, dopo qualche giorno dall'inserzione, una volta organizzato il tessuto fibroso pericannulare, rappresenta un valido ancoraggio, atto a prevenire gli strappi accidentali, ma non impedisce la rimozione da effettuare con una forte trazione qualora il catetere debba essere sostituito o rimosso. Infine per i casi particolari, è stato previsto un ancoraggio mobile da fissare alla cannula dopo aver controllato l'esatto punto nel sottocute nel quale dovrà essere allocato.

In complesso i fattori elencati ci sembrano illustrare le motivazioni che hanno indotto a progettare questo sistema che dal 1989, data della prima pubblicazione (18) dei nostri risultati con il posizionamento chirurgico di due cateteri preparati in sede, e poi nel 1990 (19) con cateteri non più preparati artigianalmente e impiantati per via per cutanea, ha dato i migliori risultati (20) non solo al nostro gruppo (10-13). Per non citare la ricca letteratura prodotta dai ricercatori italiani ho ricordato solo alcune pubblicazioni internazionali per una delle quali, il sistema Tesio Cath (11) ha avuto la copertina dell'American Journal of Kidney Disease.

\section{CONCLUSIONI}

Pur restando la FAV il "golden standard" degli accessi vascolari per emodialisi, un catetere con un lungo tunnel sottocutaneo può essere l'unica e ultima possibilità di accesso permanente ai vasi anche se non rappresenta la soluzione ideale, ma solo quella non altrimenti evitabile.

La cateterizzazione della vena giugulare interna omolaterale con due cateteri in Spi-silicone o poliuretano BioFlex inseriti e posizionati autonomamente rappresenta una sicura ed affidabile alternativa. Sono in grado di provvedere un più alto e costante flusso con minor ricircolo ematico rispetto ai cateteri monocannula per i quali gli stessi valori di adeguatezza dialitica richiedono tempi più lunghi di dialisi.

I risultati nel lungo periodo sono legati alla progettazione dei cateteri, all'appropriato posizionamento in modo da impedire il kinking, alla tunnellizzazione lunga almeno $6 \mathrm{~cm}$ parallela allo sterno, al sistema di ancoraggio ed alle caratteristiche di biotolleranza dei materiali componenti le cannule. Fondamentale è l'appropriato e meticoloso programma di gestione dei CVC da parte del personale infermieristico che, promuovendo l'educazione del paziente al tipo di accesso e uniformando il protocollo adottato dall'intero staff del Reparto, ne garantisce la durata e l'efficienza.

In attesa di nuovi materiali, i cateteri in Spi-silicone hanno dimostrato l'ottima stabilità e biocompatibilità nel tempo. I cateteri in poliuretano (BioFlex) migliorano il rapporto fra diametro interno ed esterno e, a parità di flusso, occupano uno spazio minore nella vena. Se confermata la lunga stabilità chimica e biologica del poliuretano, saranno la scelta di elezione. Nel corso di questi 18 anni altre importanti aziende hanno seguito il nostro stesso percorso (Quinton e Bellco principalmente) e ho rilevato, scorrendo la letteratura mondiale, 196 pubblicazioni riferibili all'uso dei cateteri di Tesio. In particolare ritengo importante segnalare il già citato lavoro di Powelson della Harvard Medical School di Boston (10) che nel 1996, dopo una lunga esperienza ed aver rivisto 137 articoli a riguardo ha scritto : «... In my hands, the twin catheter system has minimized these difficulties and has therefore become my preferred choice. Please note that I have no financial or commercial interest in the company making these catheters; my 


\section{Giornale \\ di Tecniche \\ Nefrologiche \\ \& Dialitiche \\ Anno XVIII n ${ }^{\circ} 4$ \\ (C) Wichtig Editore 2006

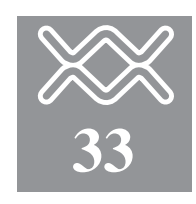

preference is based strictly on review of the literature, the intraoperative feel of the twin cath and the savage of difficult patients». E l'articolo nel 2004 di Duncan et al del St. Mary Hospital di Londra (13) riguardante 623 impianti di cateteri di Tesio : «Tesio Caths provide effective and safe longterm vascular access».

Il catetere di Tesio è maggiorenne!

\section{BIBLIOGRAFIA}

1. Pisoni RL, Young EW, Dykstra $\mathrm{DM}$, et al. Vascular access use in Europe and United States Results from the DOPPS. Kidney Int 2002; 61: 305-16.

2. Pastan S, Soucie JM, McClellan WM. Vascular access and increased risk of death among hemodialysis patients. Kidney Int 2002; 62: 620-6.

3. Guillaume J, Charra JB, Chazot $\mathrm{C}$, et al. Long-term outcome of permanent hemodialysis catheters: A Controlled Study. Blood Purif 2001; 19: 401-7.

4. Canaud B, Beraud JJ, Joyeux H, Mion C. Internal jugular vein cannulation using 2 silastic catheters. $\mathrm{Ne}$ phron 1986; 43: 133-8.

5. Cimochowski GE, Worley E, Rutherford WE, et al. Superiority of the internal jugular vein over the subclavian access for temporary dialysis. Nephron 1990; 54: 154-61.

6. Schillinger F, Schillinger D, Montagnac R, Milcent T. Post catheterisation vein stenosis in hemodialysis: Comparative angiographic study of 50 subclavian end 50 internal jugular access. Nephrol Dial Transplant 1991; 6: 722-4.

7. Uldall PR. Subclavian cannulation is no longere necessary or justified in patients with end-stage renal failure. Semin Dial 1994; 7: 161-4.

8. Konner K. Subclavian haemodialysis access: is it still justified in 1995? Nephrol Dial Transplant 1995. Editorial Comments.

9. Tesio F, DeBaz H, Panarello G, et al. Double catheterization of the internal jugular vein for hemodialysis: Indications, technique and clinical results. Artif Organs 1994, 18: 301-4.

10. Powelson JA. New techniques and catheters for long-term Central Venous Access. Acta of the New England Joint Vascular MTG. 26 April, 1996.
11.

Prakash N, Prabhu MD, Scott R, et al. Long term performance and complications of the Tesio Twin $\mathrm{Ca}$ theter System for Hemodialysis Access. Am J Kidney Dis 1997; 2: 213 8 .

12. Wivell W, Bettmann A, Baxter B, et al. Outcomes and performance of the Tesio Twin Catheter System placed for Hemodialysis Access. Radiology 2001; 3: 697-703.

13. Duncan NDC, Singh P, Cairns HD, et al. Tesio caths provide effective and safe long term vascular access. Nephrol Dial Transplant 2004;11: 2816-22.

14. Galli F, Mandolfo S. Il CVC come accesso vascolare per emodialisi. In: Gessaroli M: Accessi Vascolari per Emodialisi. Ed. Dompé Biotec 2001; 3-34.

15. De Cicco M, Panarello G, Tesio F, et al. Source and route of microbial colonization of parenteral nutrition catheters. Lancet 1989; 25: 1258-61.

16. Sioshansi P. New processes for Surface Treatment of Catheters. Artif Organs 1994; 18 (4): 266-71.

17. Bambauer R, Mestres P, Pirrung KJ, Sioshansi P. Scanning Electron Microscopic Investigation of Catheters for Blood Access. Art Organs; 1994, 18(4): 272-5

18. Tesio F, Calianno G, Cecchin E, et al. Il Cateterismo della vena giugulare interna con due cateteri in silastic per il trattamento emodialitico cronico. Atti del II Congresso Int. sulle Protesi Vascolari. Monduzzi Ed. Bologna, 1989: 165-9.

19. Tesio F, Callianno G, Panarello G. et al. Il cateterismo della vena giugulare interna per il trattamento emodialitico acuto e cronico. Nefrologia, Dialisi e Trapianto. Witchig Ed. Milano, 1990; 31: 1107-8.

20. Tesio F, De Baz H, Panarello G. Successful Long-term Central Venous Access. Home Hemodialysis Int 1998; 2: 38-40. 